Richard Bryant ORCID iD: 0000-0002-9607-819X

Leanne Williams ORCID iD: 0000-0001-9987-7360

\title{
Differential Neural Predictors of Treatment Response for Fear and Dysphoric Features of Posttraumatic Stress Disorder
}

Richard A. Bryant, $\mathrm{PhD}^{1,2}$, May Erlinger, $\mathrm{BSc}^{2}$, Kim Felmingham $\mathrm{PhD}^{3}$, Gin $\mathrm{S}$. Malhi, $\mathrm{MD}^{4}$, Meaghan L. O’Donnell, $\mathrm{PhD}^{5}$, Leanne M. Williams, $\mathrm{PhD}^{6,7}$, \& Mayuresh S. Korgaonkar, $\mathrm{PhD}^{2,8}$

1. School of Psychology, University of New South Wales.

2. Brain Dynamics Centre, Westmead Institute for Medical Research, University of Sydney.

3. Discipline of Psychological Science, University of Melbourne

4. Department of Psychiatry, University of Sydney

5. Phoenix Australia Centre for Posttraumatic Mental Health, University of Melbourne

6. Department of Psychiatry and Behavioral Sciences, Stanford University

7. Sierra-Pacific Mental Illness Research, Education, and Clinical Center (MIRECC) VA Palo Alto Health Care System, Palo Alto

8. School of Health Sciences, Faculty of Medicine and Health, University of Sydney

* Equal senior authors

\section{CORRESPONDING AUTHOR}

Richard Bryant

School of Psychology

This is the author manuscript accepted for publication and undergone full peer review but has not been through the copyediting, typesetting, pagination and proofreading process, which may lead to differences between this version and the Version of Record. Please cite this article as XXXX.

This article is protected by copyright. All rights reserved. 
University of New South Wales

Sydney, NSW 2052

Tel: 61-2-93853640

Fax: 61-2-93853641

r.bryant@unsw.edu.au

Key Words: posttraumatic stress disorder; cognitive behavior therapy; neuroimaging; treatment resistance; trauma.

RUNNING TITLE: NEURAL PREDICTORS OF PTSD TREATMENT

Abstract

Backgrounds: Although trauma-focused cognitive behavior therapy (TF-CBT) is the frontline treatment for posttraumatic stress disorder (PTSD), at least one-third patients are treatment non-responders. This study aimed to identify neural markers of treatment response, and specifically prediction of remission of specific PTSD symptoms.

Methods: This study assessed PTSD treatment-seeking patients $(n=40)$ prior to TFCBT during functional magnetic brain resonance imaging (fMRI) when they processed fear, sad, happy, and neutral faces. Patients underwent 9 sessions of TFCBT, and were independently assessed on the Clinician-Administered PTSD Scale following treatment. Treatment responders and non-responders were compared with healthy controls $(n=40)$. Severity of PTSD was assessed with the ClinicianAdministered PTSD Scale. FMRI responses were calculated for each emotion face compared to neutral contrast, which were correlated with reduction of PTSD severity

This article is protected by copyright. All rights reserved. 
from pretreatment to posttreatment. Treatment response was categorized by at least $50 \%$ reduction in severity of PTSD.

Results: Left insula activation during processing of both sad and fear faces was associated with greater reduction of fear, but not dysphoric, symptoms after treatment. Connectivity of the left insula to the pregenual anterior cingulate cortex was associated with poorer response to treatment. Responders and controls had similar levels of activation and connectivity, and were different from non-responders. Conclusions: Positive response to TF-CBT is predicted during emotion processing by normal levels of recruitment of neural networks implicated in emotional information. These findings suggest that distinct neural networks are predictive of PTSD fear and dysphoric symptom reduction following TF-CBT.

Trial Registration: Prospectively registered at Australian and New Zealand Clinical Trials Registry, ACTRN12612000185864 and ACTRN12609000324213.

Although trauma-focused cognitive behavior therapy (TF-CBT) is the preferred treatment for PTSD, one-third of patients do not respond to this approach (Bradley, 2005). Increasing attention is being given to neural $\mathrm{m}$ rkers that may identify patients who are non-responsive to CBT. Most of these studies have used functional magnetic resonance imaging (fMRI) prior to treatment. These studies have found that better treatment response is associated with decreased ventral anterior cingulate activation during nonconscious fear processing (Bryant et al., 2008a), increased activation of the dorsal anterior cingulate in anticipation of negative images (Aupperle et al., 2013), reduced dorsal anterior cingulate activation during image presentation (Aupperle et al., 2013; van Rooij, Kennis, Vink, \& Geuze, 2016), 
reduced amygdala activity to nonconscious (Bryant et al., 2008a) and conscious (van Rooij et al., 2016) processing of negative stimuli, increased left inferior parietal lobe activation during contextual cue processing (van Rooij, Geuze, Kennis, Rademaker, \& Vink, 2015), and greater activation of the dorsal prefrontal cortex and reduced amygdala activation during emotion processing, and greater ventromedial/ventral striatal activation during emotion conflict regulation (Fonzo et al., 2017).

A limitation of these neuroimaging studies is that they have used neural activation to predict clinical change only in terms of the global construct of PTSD. There is increasing recognition that the diagnostic conceptualization of PTSD is actually highly heterogeneous (Galatzer-Levy \& Bryant, 2013), and there are good reasons to expect different neural substrates to predict how different types of PTSD patients may respond to treatment. For example, PTSD patients with more dissociative responses tend to overmodulate emotions, involving prefrontal inhibition of limbic regions, relative to those with more fear and hyperarousal responses (Felmingham et al., 2008; Lanius et al., 2010). Many factor analytic studies of PTSD have identified four factors that include re-experiencing, avoidance, arousal, and either numbing or dysphoric symptoms (Armour, O'Connor, Elklit, \& Elhai, 2013; King, Leskin, King, \& Weathers, 1998; Elhai and Palmieri 2011). Studies have shown that these four factors can be explained as forming two latent factors that comprise fear (including reexperiencing, active avoidance, hypervigilance, and elevated startle) and dysphoric (passive avoidance, sleep disturbance, concentration difficulties, and irritability) symptoms (Forbes et al., 2010, 2015). These latent symptom constructs may represent a neurobiologically relevant means to measure treatment response 
because they may map onto distinct neural networks that are evoked by stimuli that represent equivalent constructs. This proposal is supported by several converging lines of evidence. First, fear stimuli (such as facial expressions of fear) relevant to the construct of Fear symptoms have been shown reliably to engage amygdala-dorsal ACC and dorsal medial prefrontal networks under conditions of explicit processing and amygdala-ventral ACC networks under nonconscious conditions of implicit processing, and to elicit distinct modes of functional connectivity in these networks during explicit and implicit fear processing (Robinson et al., 2014). Patients with PTSD exhibit abnormal profiles of activation in these networks when probed with the same fear stimuli, including amygdala hyper-reactivity and hypo-reactivity depending on level of awareness (Bryant et al., 2008b). Second, sad stimuli relevant to the construct of Dysphoria symptoms have also been found to engage the amygdala and ventral ACC. Third, happy face stimuli also relevant to the construct of Dysphoria symptoms have been found to engage regions central to the positive affect, or reward, circuits of the human brain (Whitton, Treadway, \& Pizzagalli, 2015). In PTSD, we have observed reduced activation of these same regions when probed by happy face stimuli and reduced reward-related striatal and subcortical connectivity (Felmingham et al., 2014; Zhu et al., 2017).

In the context of evidence that PTSD is characterized by two distinct biologically relevant factors, this study aimed to advance our understanding of neural predictors of TF-CBT for PTSD by using valenced facial stimuli to probe negative and positive affect circuits, and to quantify the contribution of these circuits as prognostic biomarkers for reduction of fear and dysphoric symptoms. PTSD patients 
viewed fear, sad and happy faces to determine the extent to which activation of emotional networks was associated with reductions in fear and dysphoric symptoms. We hypothesized that activation and connectivity within regions central to the negative affect circuitry (i.e. the amygdala, hippocampus, ventral anterior cingulate cortex and insula) probed by fear and sad faces would be prognostic of a subsequent post-treatment reduction in PTSD fear symptoms, whereas regions central to positive affect and reward circuitry (i.e. the amygdala, dorsal anterior cingulate, striatal cortex and medial orbitofrontal cortex) probed by happy faces would be prognostic of a posttreatment reduction in dysphoric symptoms of PTSD.

\section{Materials and Methods}

\section{Participants}

Participants were 50 treatment-seeking patients, enrolled into one of two treatment trials between 2011 and 2016 (Bryant et al., 2013, 2018); 40 of these patients were included in the imaging analyses (with 10 excluded for either failing to complete the relevant task or not returning for post-treatment assessment). Only patients randomized to the exposure therapy arms of the two trials (determined by a random number generator) were included in the current study. The mean age of the 40 patients (19 females, 21 males) was $40.60 \pm 11.3$ years, who were diagnosed with PTSD by clinical psychologists using the Clinician Administered PTSD Scale (CAPS (Blake et al., 1995)) following assault, childhood abuse, motor vehicle accidents, or

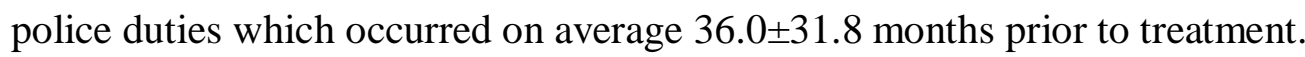
Participants with a history of neurological disorder, moderate or severe traumatic brain injury, psychosis, or substance dependence were excluded. The protocol 
permitted prescribed medication if the dosage had remained stable for two months prior to the scan and was not altered during the course of the study; 11 (27.5\%) participants were taking selective serotonin reuptake inhibitors. The study also included a comparison group of 40 healthy participants (18 females, 22 males) of mean age 39.2 \pm 15.8 years who had never experienced a criterion A stressor and with no current Axis I disorders, as assessed by the Mini International Neuropsychiatric Interview (MINI version 5.5) (Sheehan et al., 1998). Depression and anxiety levels for patients were also assessed by self-report using the Depression, Anxiety, and Stress Scale (DASS; Lovibond \& Lovibond, 1995). Participant characteristics are described in Table 1.

\section{Procedure}

The study was approved by the Western Sydney Area Health Service Human Research Ethics Committee and written informed consent was obtained from participants. Participants were initially assessed for PTSD (as defined by DSM-IV) by clinical psychologists using the CAPS and for comorbid Axis I disorders, the MINI was used to assess for current major depressive episode (MDE), generalized anxiety disorder, social phobia, panic disorder, agoraphobia, obsessive-compulsive disorder, and a substance use disorder.

\section{Imaging Procedure}

During the fMRI session, participants completed two viewing of facial expressions of emotion tasks, one designed to probe explicit appraisal of emotion stimuli and the other to probe nonconscious reactivity to the same stimuli. These tasks 
have been established previously (Bryant et al., 2008), and demonstrated to be relevant for predicting treatment outcomes in relevant clinical disorders (GoldsteinPiekarski et al., 2016; Williams et al., 2015). Briefly, the patients either passively viewed a series of emotional faces (angry, disgust, fear, sad, happy, and neutral) for 500ms with an interstimulus interval of 750ms (explicit task) or viewed the same emotional faces with a stimulus duration of $10 \mathrm{~ms}$, immediately followed by a neutral face for 150ms, and an interstimulus interval of 1100ms (implicit task) (see Supplementary Materials for imaging protocol).

\section{Treatment protocol}

Within 2-3 weeks of scanning, participants commenced a course of 9 onceweekly individual sessions of TF-CBT that were delivered by experienced doctorallevel or masters-level clinical psychologists. This therapy involved an initial session of psychoeducation about psychological responses to trauma, then 6 sessions of 40minute imaginal exposure to the trauma memory, instructions regarding in vivo exposure to avoided situations, and cognitive restructuring of thoughts related to the traumatic event. An additional session reinforced cognitive restructuring exercises, and a final session focused relapse prevention (Bryant et al., 2013, 2018). This therapy procedure is consistent with gold standard TF-CBT protocols (McLean, Asnaani, \& Foa, 2015). Independent clinicians rated the fidelity of 130 sessions (18\%), indicating full adherence to the treatment protocols and high level of quality on a 7 -point scale $($ mean $=6.11, S D=1.32)$. There were no treatment effects according to different therapist or therapist qualifications. A posttreatment assessment

This article is protected by copyright. All rights reserved. 
using the CAPS was conducted by an independent clinical psychologist one week following completion of the course of treatment.

\section{Data Analyses}

The fMRI preprocessing, models and connectivity analysis details for this task have been previously published and included in the supplementary material. To test the hypothesized symptom constructs and as done in previous emotional processing fMRI studies, contrast images were derived for FEAR versus NEUTRAL, SAD versus NEUTRAL and HAPPY versus NEUTRAL trials. To define the circuits of focus for this study we used a region of interest (ROI) approach motivated by a synthesis of existing knowledge, grounded in meta-analytic studies (Williams, 2016). To test the specific hypotheses regarding neural activation associated with emotional processing of negative emotion (FEAR and SAD faces), we employed the use of the negative affect network (comprising of the subgenual [sgACC] and pregenual anterior cingulate cortex, [pgACC], bilateral amygdala, insula, and hippocampus), chosen for its relevance to neural circuitry of negative emotion processing (Kober et al., 2008). To test hypotheses regarding neural activation associated with positive emotion processing (HAPPY faces), we employed the use of the positive affect network (comprising of the medial orbitofrontal cortex [mOFC], dorsal anterior cingulate cortex [dACC], bilateral amygdala, and bilateral striatum [caudate, putamen, and pallidum]), chosen for its relevance to neural circuity of positive emotion processing (Liu, Hairston, Schrier, \& Fan, 2011).

All ROI voxel-wise statistical analyses listed below were conducted at a family wise error corrected p-level of 0.05 (pFWE<0.05), corrected for multiple 
comparisons by inputting the various ROIs as a single mask at this threshold. To examine change in PTSD severity independent of initial severity (in a manner that allows comparison with prior studies of prediction of treatment response), residual change was calculated from a regression of pre-treatment total CAPS scores on posttreatment total CAPS scores (Siegle, Carter, \& Thase, 2006) (higher change scores correspond to greater improvement), and were correlated with neural measures. To examine change in PTSD severity, whilst considering the heterogeneity of the disorder, residual change was calculated from a regression of pre-treatment CAPS factor scores (Fear factor scores and Dysphoria factor scores) on post-treatment CAPS factor scores, and were correlated with neural measures. Fear factor was defined as total score of reexperiencing, active avoidance, hypervigilance, and elevated startle symptoms and dysphoric factor as a total score of passive avoidance, sleep disturbance, concentration difficulties, and irritability symptoms (Forbes et al., 2010). These residual PTSD severity scores were then correlated voxel-wise within our selected ROI for each emotion contrasts to identify associations between pretreatment neural activity with treatment recovery.

\section{Functional connectivity analyses}

Next, we conducted functional connectivity analyses (generalized psychophysiological interactions [gPPI]) (McLaren, Ries, Xu, \& Johnson, 2012) using significant clusters from the activation correlation analyses to determine whether neural connectivity for the relevant seeds was similarly associated with treatment response (overall change in CAPS, and individual factor change scores). We evaluated connectivity related to significant contrasts from the activation 
correlation analyses in second level ROI analyses testing for voxel-wise correlations between connectivity and residual PTSD severity as done for activation previously (see Supplementary Materials).

\section{Comparison of PTSD symptom response outcomes relative to healthy controls}

Next, we categorized the PTSD cohort into treatment responders or nonresponders (responders defined as those with at least 50\% reduction in pre-treatment CAPS) to evaluate baseline neural activation and connectivity profiles of responders and non-responders relative to healthy controls. Mean beta values for the significant clusters from the voxelwise analyses above (i.e. pretreatment activation and connectivity associations with change in PTSD severity) were extracted and analysis of variance (ANOVA) analyses were performed using the three groups followed by post-hoc group contrasts using the Bonferroni corrected $\mathrm{p}<0.05$ threshold. We also determined whether the significant neural measures predicted treatment response above and beyond clinical and demographic variables. We employed cross-validation statistics to test generalizability of results and to identify the best predictive models (supplementary materials).

\section{Exploratory analyses}

We also performed exploratory whole brain voxelwise correlation analyses to test any effects beyond our hypothesized ROIs (see Supplementary Analyses). A series of additional supplementary analyses were also conducted: 1) to evaluate group specific activations and group differences in neural activations for emotional contrasts for the PTSD and healthy control groups as voxel wise single and two sample t-tests. 
We also evaluated effects for the NEUTRAL vs. implicit baseline "REST" condition to rule out if effects were driven by the neutral condition; 2) to evaluate generalizability to further samples and to determine accuracy, sensitivity, and specificity of the measures in predicting treatment response (responders vs nonresponders) using cross-validation analyses, as a general model and as a bestpredictive fit model; 3) to determine whether the significant neural measures predicted treatment response above and beyond clinical and demographic variables; and 4) to evaluate any confounds due to medication use on neural measures.

\section{Results}

\section{Clinical Outcomes}

All 40 included participants met DSM-IV criteria for PTSD prior to treatment and completed 7-9 sessions of TF-CBT. The mean CAPS score was $71.8 \pm 17.6$ at pretreatment and 28.1 \pm 20.0 following TF-CBT treatment. Based on our set criteria, 27 (67.5\%) participants classified as treatment responders and 13 (32.5\%) were classified as non-responders. Participant characteristics are outlined in Table 1.

\section{Association Between Symptom Change and Pre-treatment Neural Measures}

Significant correlations between PTSD symptom improvement (reduction in CAPS scores) and pretreatment neural measures were observed for explicit presentations of sad and fearful faces. No significant associations were observed for explicit processing of happy faces. Similarly, there were no significant associations for implicit presentations. For sad vs neutral, a significant positive correlation was found between reduction in overall PTSD severity and left insula activation (Table 2;

This article is protected by copyright. All rights reserved. 
Figure 1A). There was similarly a significant positive correlation between reduction in PTSD fear symptoms and left insula activation for both sad and fearful faces (Table 2, Figure 1B) but no significant voxelwise correlations for reduction in PTSD dysphoric symptoms and activation (additional analyses were conducted on wholebrain analyses, as well as analyses of single sample activations; see Supplementary Tables S1, S2 and S3).

For sad vs neutral faces, connectivity of the left insula to the pgACC was significantly negatively correlated with a reduction in overall PTSD severity, PTSD fear symptoms and PTSD dysphoria symptoms (Table 2; Figure 2).

These effects were not driven by the neutral condition (all $\mathrm{p}>0.05$ for NEUTRAL vs REST). These effects were all significant controlling for medications (Supplementary Tables S4 and 5). To identify whether low baseline symptom scores or differences in DASS anxiety scores affected significant results, these results were re-analyzed controlling for baseline symptom severity (as an overall CAPS change score, and individual factor change scores) and then DASS anxiety; all originally observed effects remained significant. To determine the power of our sample $(n=40)$ for detecting the observed effects, a post-hoc power analysis indicated that the achieved power was $47.7 \%$ for low (Cohen's $d=0.3)$ and $92.2 \%$ for medium $(d=0.5)$ effect sizes; suggesting there was limited power to detect small to medium effect sizes.

This article is protected by copyright. All rights reserved. 
Comparison of neural profiles between treatment responders, non-responders, and healthy controls

The main effect of group was found significant for all the neural activation and connectivity measures that were associated with PTSD symptom improvement described above (see Table 3). Posthoc analyses revealed that across all measures, treatment non-responders had significantly less activation but more connectivity than healthy controls and responders. Responders on the other hand had similar pretreatment levels of activation and connectivity relative to controls.

\section{Predictive models of treatment response}

In attempting to illustrate how the neural markers we identified could be helpful for treatment decisions and informing future studies, we evaluated how the predictive value of the significant neural measures compared to demographic and clinical variables, the generalizability of the models using cross-validation statistics and identifying the best predictive model to predict treatment response.

In this cohort, the demographic and clinical measures model alone did not predict treatment response. However, the inclusion of the neural measures significantly improved its predictive values $(p=0.001)$. Cross-validated linear regression analyses of a general model using mean fMRI signal from all predefined network ROIs (without any bias of our main analysis findings), replicated to some extent the correlation findings from the main analysis described above (Supplementary Tables S6 and S7). The best predictive model in classifying responders and non-responders, using the significant neural measures identified in the 
main analyses, identified left insula activation to sad faces, correlated to a change in Fear factor scores, to be the best predictive feature of our analyses (Supplementary Table S8).

Discussion

This study represents the first investigation of neural processes prior to TFCBT predicting treatment response on different features of PTSD. Overall PTSD symptom severity reduction was associated with greater activation of the left insula when participants were processing sad faces. During processing of both sad and fear faces, better treatment response was positively associated with pre-treatment activation of the left insula in terms of reduction of fear but not dysphoric symptoms. Further, greater connectivity of the left insula to the pgACC was associated with poorer response to treatment (i.e. reduced symptom reduction) in terms of overall PTSD, as well as both fear and dysphoric symptoms.

The finding that greater symptom reduction following TF-CBT was associated with activation of the left insula accords with the proposal that successful response to exposure-based treatments involves sufficient emotional processing (Foa, 2006). It is noteworthy that the co-ordinates in the current study involved the more anterior portion of the insula, which has been repeatedly associated with affective processing (Uddin, Nomi, Hebert-Seropian, Ghaziri, \& Boucher, 2017). The current finding suggests that the capacity to engage this affective processing region during processing of negative emotions may be an important predictor of positive response to TF-CBT, arguably because it reflects the capacity to engage emotions. This interpretation needs to be understood in the context of previous studies that have reported that response to

This article is protected by copyright. All rights reserved. 
TF-CBT is associated with reduced activation of the salience network (Bryant et al., 2008; van Rooij et al., 2016). These findings have been interpreted in terms of the insula, and related salience regions, been hyperactivated during affective processing, which reflects the inability to regulate emotions and are therefore prognostic of poor outcome (Colvonen et al., 2017). This interpretation may depend, however, on the nature of the stimulus presented and how this may engage emotion processing networks. For example, van Rooij et al.’s (2016) finding that insula activation in response to viewing negative images was associated with poorer response to TF-CBT may be attributed to their use of images depicting negative and even traumatic scenes; in contrast, the current study used faces with different emotional expressions. It is possible that whereas activating the insula during more generic emotional processing may reflect a capacity for emotional engagement that is optimal for TF-CBT, overactivation of the insula when processing more salient and even traumatic stimuli may reflect over-reactivity of the salience network that may be prognostic of poor treatment response.

A particularly novel aspect of this study is the demonstration that during processing of fear and sad faces activation of the left insula was predictive of reduction of fear rather than dysphoric symptoms of PTSD. Increasing evidence indicates that PTSD is a multifaceted disorder that extends beyond the construct of fear (Elhai \& Palmieri, 2011). It is relevant that prevailing models of PTSD focus on the fear circuity of neural networks, including the salience detection network, with the presumption that these neural circuits are relevant to processing of fear. The finding that insula activation predicted reduction of fear, rather than dysphoric, symptoms 
highlights that specific neural circuits may be implicated in the remission of different features of PTSD recovery. In the context of evidence of dysphoric symptoms of PTSD predicting poor response to psychotherapy (Bryant, Moulds, Guthrie, Dang, \& Nixon, 2003; Schumm, Dickstein, Walter, Owens, \& Chard, 2015), these results suggest that there is a need to understand not only the neural factors predicting reduction in fear symptoms but also those implicated in dysphoric PTSD presentations.

The observation that diminished symptom reduction was associated with connectivity of the left insula to the pgACC needs to be considered in the context of the role of the pgACC. The pgACC is situated between the dorsal ACC, which is implicated in error detection and working memory, and the ventral ACC, which involves emotion processing, salience detection, and emotion regulation (Bush, Luu, \& Posner, 2000). Whereas hyperactivation of the pgACC predicts better antidepressant response for depression (Chen et al., 2007; Davidson, Irwin, Anderle, \& Kalin, 2003), psychotherapy trials of depression have found reduced activation of the ACC being associated with symptom reduction (McLaren et al., 2012; Siegle et al., 2006). It has been suggested that CBT approaches may optimize regulatory networks (such as the pgACC) in patients who are less likely to engage these circuits naturally (DeRubeis, Siegle, \& Hollon, 2008). Given the role of the insula in emotional processing, it is possible that reduced connectivity between the pgACC and insula whilst processing negative emotions reflects less need to engage regulatory and affective networks because these patients are more able to manage affective states and therefore respond positively to exposure-based therapy. 
It is worth noting that no predictive effects were found for implicit processing of negative stimuli. This contrasts with one earlier finding from a small pilot study that found that greater amygdala activation during implicit viewing predicted poor response to TF-CBT (Bryant et al., 2008). A central tenet of TF-CBT is that PTSD patients can access emotional memories to a sufficient degree to allow regulation of distress and integration of new corrective information (Foa, 2006). It is possible that the finding of increased insula activation predicting better treatment response, and particularly in terms of reducing fear symptoms, reflects the capacity of patients to engage emotions under controlled processing; in contrast, automatic processing of fear during may not be indicative of intentional engagement of aversive emotions.

We note several methodological limitations. First, we did not include a PTSD wait-list condition that would have provided a stricter index of the predictive capacity of baseline neural responses on TF-CBT relative to spontaneous remission; it is noteworthy, however, that spontaneous remission is unlikely in the sample for whom the mean time since trauma exposure was three years. Second, replication with a larger sample size would permit stronger comparisons between treatment responders and non-responders, and closer investigation of subtypes of PTSD. Third, we note that a proportion of participants were using SSRIs; the dosage was stable for two months prior to scanning and secondary analyses indicated that all significant results were observed in non-medicated participants. Fourth, prediction of symptom reduction at longer-term follow-up assessments would provide more insight into how neural responding at baseline is related to long-term remission, and possibly relapse.

This article is protected by copyright. All rights reserved. 
In the context of increasing recognition that PTSD is a heterogeneous disorder that encompasses features beyond fear circuitry, there is a need for more precise predictors of targeted symptom reduction in TF-CBT. The current findings indicate that whereas activation of threat detection regions during processing of negative emotions (i.e. insula) predict reduction of fear symptoms in PTSD, they are not predictive of dysphoric symptoms. This finding accords with well-documented neural networks in fear circuitry models of PTSD, highlights that future studies that identify neural predictors of treatment outcome could usefully delineate between fear and dysphoric features of the disorder.

\section{Acknowledgements}

This research was supported by a NHMRC Program Grant (1073041), NHMRC Centre for Clinical Research Excellence in Anxiety (1023043), and the Centenary of Anzac Centre, a Department of Veterans’ Affairs funded initiative of Phoenix Australia.

\section{Conflicts of Interest}

The authors declare no conflict of interest.

\section{Data Sharing Statement}

Data available on request from the authors but under embargo until further analyses completed.

This article is protected by copyright. All rights reserved. 


\section{References}

Armour, C., O'Connor, M., Elklit, A., \& Elhai, J.D. (2013). Assessing posttraumatic stress

disorder's latent structure in elderly bereaved European trauma survivors: evidence for a five-factor dysphoric and anxious arousal model. Journal of Nervous and Mental Disease, 201, 901-906.

Aupperle, R. L., Allard, C. B., Simmons, A. N., Flagan, T., Thorp, S. R., Norman, S. B.,... Stein, M. B. (2013). Neural responses during emotional processing before and after cognitive trauma therapy for battered women. Psychiatry Research, 214(1), 48-55.

Blake, D. D., Weathers, F., Nagy, L. M., Kaloupek, D. G., Gusman, F. D., Charney, D. S., \& Keane, T. M. (1995). The development of a clinician administered PTSD scale. Journal of Traumatic Stress, 8(1), 75-90.

Bradley, R. (2005). A multidimensional meta-analysis of psychotherapy for PTSD (vol 162, pg 214, 2005). American Journal of Psychiatry, 162(4), 832-832.

Bryant, R. A., Felmingham, K., Kemp, A., Das, P., Hughes, G., Peduto, A., \& Williams, L. (2008). Amygdala and ventral anterior cingulate activation predicts treatment response to cognitive behaviour therapy for post-traumatic stress disorder. Psychological Medicine, 38(4), 555-561.

Bryant, R. A., Kemp, A. H., Felmingham, K. L., Liddell, B., Olivieri, G., Peduto, A.,... Williams, L. M. (2008). Enhanced amygdala and medial prefrontal 
activation during nonconscious processing of fear in posttraumatic stress disorder: an fMRI study. Human Brain Mapping, 29(5), 517-523.

Bryant, R. A., Kenny, L., Rawson, N., Cahill, C., Joscelyne, A., Garber, B.,... Nickerson, A. (2018). Efficacy of exposure-based cognitive behaviour therapy for post-traumatic stress disorder in emergency service personnel: a randomised clinical trial. Psychological Medicine, 1-9.

Bryant, R. A., Mastrodomenico, J., Hopwood, S., Kenny, L., Cahill, C., Kandris, K., \& Taylor, K.. (2013). Augmenting cognitive behavior therapy for PTSD with emotion tolerance training: a randomized controlled trial. Psychological Medicine, 43, 2153-2160.

Bryant, R. A., Moulds, M. L., Guthrie, R. M., Dang, S. T., \& Nixon, R. D. V. (2003). Imaginal exposure alone and imaginal exposure with cognitive restructuring in treatment of posttraumatic stress disorder. Journal of Consulting \& Clinical Psychology, 71(4), 706-712.

Bush, G., Luu, P., \& Posner, M. I. (2000). Cognitive and emotional influences in anterior cingulate cortex. Trends in Cognitive Science, 4(6), 215-222.

Chen, C. H., Ridler, K., Suckling, J., Williams, S., Fu, C. H., Merlo-Pich, E., \& Bullmore, E. (2007). Brain imaging correlates of depressive symptom severity and predictors of symptom improvement after antidepressant treatment. Biological Psychiatry, 62(5), 407-414.

This article is protected by copyright. All rights reserved. 
Davidson, R. J., Irwin, W., Anderle, M. J., \& Kalin, N. H. (2003). The neural substrates of affective processing in depressed patients treated with venlafaxine. American Journal of Psychiatry, 160(1), 64-75.

DeRubeis, R. J., Siegle, G. J., \& Hollon, S. D. (2008). Cognitive therapy versus medication for depression: treatment outcomes and neural mechanisms. Nature Review of Neuroscience, 9(10), 788-796.

Elhai, J. D., \& Palmieri, P. A. (2011). The factor structure of posttraumatic stress disorder: a literature update, critique of methodology, and agenda for future research. Journal of Anxiety Disorders, 25(6), 849-854.

Felmingham, K., Kemp, A. H., Williams, L., Falconer, E., Olivieri, G., Peduto, A., \& Bryant, R. (2008). Dissociative responses to conscious and non-conscious fear impact underlying brain function in post-traumatic stress disorder. Psychological Medicine, 38(12), 1771-1780.

Felmingham, K. L., Falconer, E. M., Williams, L., Kemp, A. H., Allen, A., Peduto, A., \& Bryant, R. A. (2014). Reduced amygdala and ventral striatal activity to happy faces in PTSD is associated with emotional numbing. PLoS One, 9(9), e103653.

Foa, E. B. (2006). Psychosocial therapy for posttraumatic stress disorder. Journal of Clinical Psychiatry, 67, 40-45.

Fonzo, G. A., Goodkind, M. S., Oathes, D. J., Zaiko, Y. V., Harvey, M., Peng, K. K.,... Etkin, A. (2017). PTSD psychotherapy outcome predicted by brain 
activation during emotional reactivity and regulation. American Journal of Psychiatry, 174(12), 1163-1174.

Forbes, D., Lockwood, E., Elhai, J. D., Creamer, M., Bryant, R., McFarlane, A.,... O'Donnell, M. (2015). An evaluation of the DSM-5 factor structure for posttraumatic stress disorder in survivors of traumatic injury. Journal of Anxiety Disorders, 29, 43-51.

Forbes, D., Parslow, R., Creamer, M., O'Donnell, M., Bryant, R., McFarlane, A.,... Shalev, A. (2010). A longitudinal analysis of posttraumatic stress disorder symptoms and their relationship with Fear and Anxious-Misery disorders: implications for DSM-V. Journal of Affective Disorders, 127(1-3), 147-152.

Galatzer-Levy, I. R., \& Bryant, R. A. (2013). 636,120 Ways to Have Posttraumatic Stress Disorder. Perspectives in Psychological Science, 8(6), 651-662.

Goldstein-Piekarski, A. N., Korgaonkar, M. S., Green, E., Suppes, T., Schatzberg, A. F., Hastie, T.,... Williams, L. M. (2016). Human amygdala engagement moderated by early life stress exposure is a biobehavioral target for predicting recovery on antidepressants. Proceedings of the National Academy of Sciences, 113(42), 11955-11960.

King, D.W., Leskin, G.A., King, L.A., \& Weathers, F.W. (1998). Confirmatory factor analysis of the clinician-administered PTSD Scale: Evidence for the dimensionality of posttraumatic stress disorder. Psychological Assessment 10, 90-96.

This article is protected by copyright. All rights reserved. 
Kober, H., Barrett, L.F., Joseph, J., Bliss-Moreau, E., Lindquist, K. \& Wager, T.D. (2008). Functional grouping and cortical-subcortical interactions in emotion: A meta-analysis of neuroimaging studies. Neuroimage, 42, 998-1031.

Lanius, R. A., Vermetten, E., Loewenstein, R. J., Brand, B., Schmahl, C., Bremner, J. D., \& Spiegel, D. (2010). A dissociative subtype of PTSD: Clinical and neurobiological evidence. American Journal of Psychiatry, 167(6), 640-647.

Liu, X., Hairston, J., Schrier, M., \& Fan, J. (2011). Common and distinct networks underlying reward valence and processing stages: a meta-analysis of functional neuroimaging studies. Neuroscience and Biobehavioral Reviews, 35(5), 1219-1236.

Lovibond, P. F., \& Lovibond, S. H. (1995). The structure of negative emotional states: comparison of the Depression Anxiety Stress Scales (DASS) with the Beck Depression and Anxiety Inventories. Behaviour Research and Therapy, 33(3), 335-343

McLaren, D. G., Ries, M. L., Xu, G., \& Johnson, S. C. (2012). A generalized form of context-dependent psychophysiological interactions (gPPI): a comparison to standard approaches. Neuroimage, 61(4), 1277-1286.

McLean, C. P., Asnaani, A., \& Foa, E.B. (2015). Prolonged exposure therapy. In U. Schnyder, \& Cloitre, M. (Ed.), Evidence based treatments for trauma-related psychological disorders (pp. 143-159). Heidelberg: Springer.

This article is protected by copyright. All rights reserved. 
Robinson, O. J., Krimsky, M., Lieberman, L., Allen, P., Vytal, K., \& Grillon, C. (2014). Towards a mechanistic understanding of pathological anxiety: the dorsal medial prefrontal-amygdala 'aversive amplification' circuit in unmedicated generalized and social anxiety disorders. Lancet Psychiatry, 1(4), 294-302.

Schumm, J. A., Dickstein, B. D., Walter, K. H., Owens, G. P., \& Chard, K. M. (2015). Changes in posttraumatic cognitions predict changes in posttraumatic stress disorder symptoms during cognitive processing therapy. Journal of Consulting and Clinical Psychology, 83(6), 1161-1166.

Sheehan, D. V., Lecrubier, Y., Sheehan, K. H., Amorim, P., Janavs, J., Weiller, E.,... Dunbar, G. C. (1998). The Mini-International Neuropsychiatric Interview (M.I.N.I.): the development and validation of a structured diagnostic psychiatric interview for DSM-IV and ICD-10. Journal of Clinical Psychiatry, 59 Suppl 20, 22-33.

Siegle, G. J., Carter, C. S., \& Thase, M. E. (2006). Use of FMRI to predict recovery from unipolar depression with cognitive behavior therapy. American Journal of Psychiatry, 163(4), 735-738.

Uddin, L. Q., Nomi, J. S., Hebert-Seropian, B., Ghaziri, J., \& Boucher, O. (2017). Structure and Function of the Human Insula. Journal of Clinical Neurophysiology, 34(4), 300-306.

This article is protected by copyright. All rights reserved. 
van Rooij, S. J., Geuze, E., Kennis, M., Rademaker, A. R., \& Vink, M. (2015). Neural correlates of inhibition and contextual cue processing related to treatment response in PTSD. Neuropsychopharmacology, 40(3), 667-675.

van Rooij, S. J., Kennis, M., Vink, M., \& Geuze, E. (2016). Predicting treatment outcome in PTSD: A longitudinal functional MRI study on trauma-unrelated emotional processing. Neuropsychopharmacology, 41(4), 1156-1165.

Whitton, A. E., Treadway, M. T., \& Pizzagalli, D. A. (2015). Reward processing dysfunction in major depression, bipolar disorder and schizophrenia. Current Opinions in Psychiatry, 28(1), 7-12.

Williams, L. M. (2016). Precision psychiatry: a neural circuit taxonomy for depression and anxiety. Lancet Psychiatry, 3(5), 472-480. doi

Williams, L. M., Korgaonkar, M. S., Song, Y. C., Paton, R., Eagles, S., GoldsteinPiekarski, A.,... Etkin, A. (2015). Amygdala Reactivity to Emotional Faces in the Prediction of General and Medication-Specific Responses to Antidepressant Treatment in the Randomized iSPOT-D Trial. Neuropsychopharmacology, 40(10), 2398-2408.

Zhu, X., Helpman, L., Papini, S., Schneier, F., Markowitz, J. C., Van Meter, P. E.,... Neria, Y. (2017). Altered resting state functional connectivity of fear and reward circuitry in comorbid PTSD and major depression. Depression and Anxiety, 34(7), 641-650.

This article is protected by copyright. All rights reserved. 


\section{Figures}

Figure 1. Pre-treatment insula activation correlated $(\mathrm{pFWE}<0.05)$ with change in overall PTSD severity (A) and in PTSD fear symptoms (B) for SAD vs NEUTRAL and FEAR vs NEUTRAL contrasts. Scatter plots showing a significant positive correlation between (A) overall symptom improvement $(\mathrm{p}<0.001)$ and $(\mathrm{B})$ fear symptom improvement $(\mathrm{p}<0.001)$ with left insula activation for sad faces, and a significant positive correlation between fear symptom improvement $(\mathrm{p}<0.001)$ with left insula activation for fearful faces.

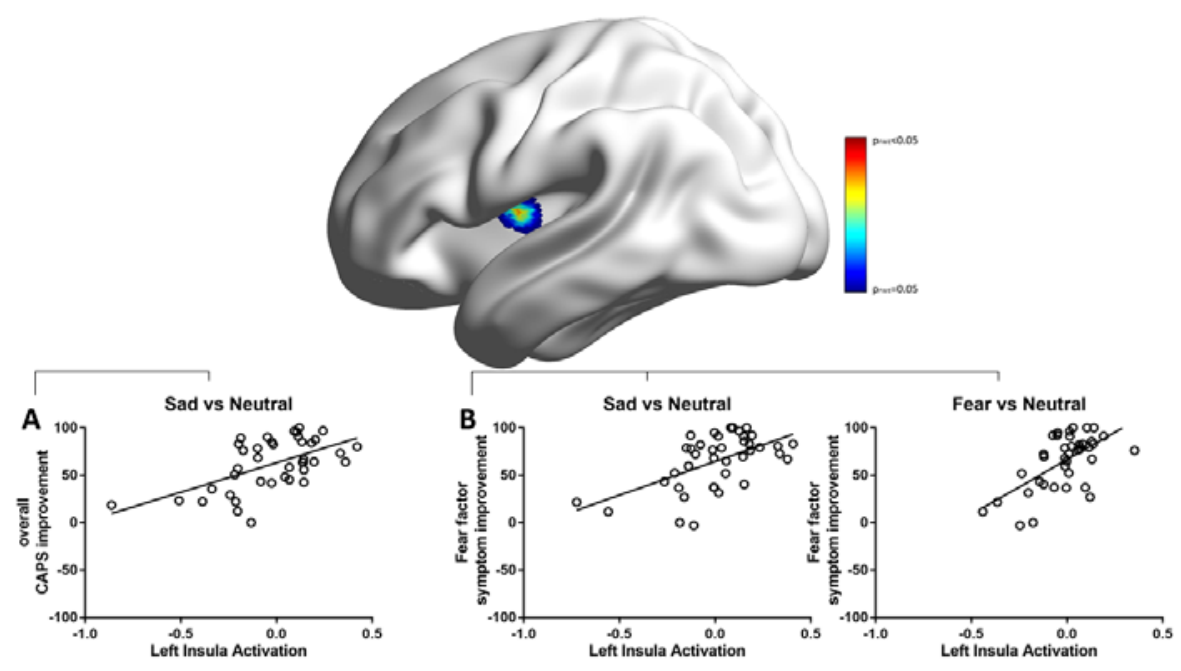

This article is protected by copyright. All rights reserved. 
Figure 2. Pre-treatment Insula-pgACC connectivity for Sad vs Neutral correlated with change in overall PTSD severity and PTSD fear symptoms. Scatter plots show significant negative correlations between overall symptom improvement ( $\mathrm{p}=0.002)$, fear symptom improvement $(\mathrm{p}<0.001)$, and dysphoria symptom improvement $(\mathrm{p}<0.001)$ with left insula to pgACC connectivity.
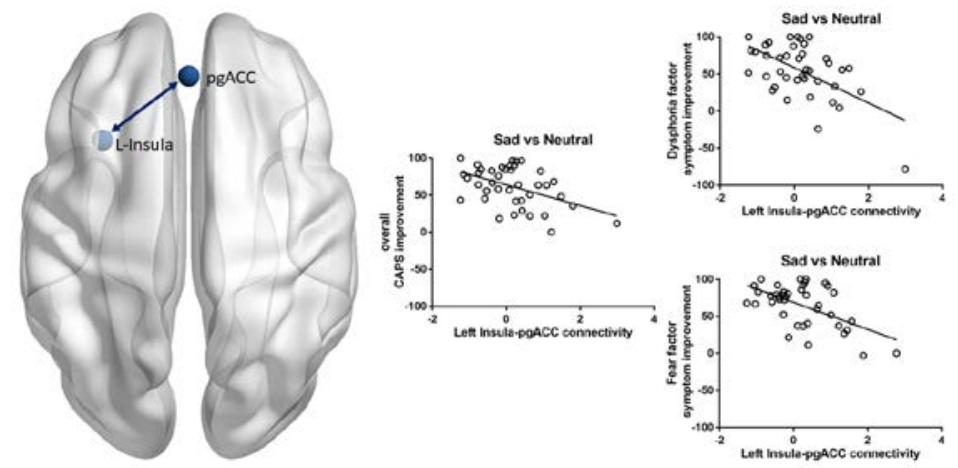

Table 1. Participant Characteristics for PTSD (Treatment Responders, NonResponders) and Healthy Controls

\begin{tabular}{|c|c|c|c|c|c|}
\hline \multirow{3}{*}{$\begin{array}{l}\text { PTSD } \\
(\mathrm{n}= \\
40)\end{array}$} & \multirow{3}{*}{$(n=40)$} & \multirow[t]{3}{*}{$\boldsymbol{T}_{78} / \chi^{2}$} & Treatment & \multirow{2}{*}{$\begin{array}{c}\text { Treatment } \\
\text { Non- } \\
\text { Responders }\end{array}$} & \multirow[t]{3}{*}{$\boldsymbol{T}_{78} / \chi^{2}$} \\
\hline & & & & & \\
\hline & & & $(n=27)$ & & \\
\hline $\begin{array}{l}40.60 \\
(11.3)\end{array}$ & $\begin{array}{c}39.2 \\
(15.8)\end{array}$ & 0.46 & 41.4 (12.7) & 39.0 (7.9) & 0.61 \\
\hline $\begin{array}{c}21 \\
(52.5)\end{array}$ & $22(55.0)$ & 0.05 & $13(48.1)$ & 8 (61.5) & 0.63 \\
\hline
\end{tabular}


Months since

Trauma, $M$

(SD)

Type of trauma,

n (\%)

Childhood

abuse

Motor

vehicle

accident

Police-

related

trauma

Assault

Prescribed

SSRI, n (\%)

Major

Depressive

Disorder, n (\%)

Social Phobia, $\mathrm{n} \quad 10$

16

11
36.0

(31.8)

$39.2(34.9) \quad 29.2(23.7)$
0.90

$4(14.8) \quad 0(0)$

5 (18.5) $3(23.1)$

7 (25.9) $\quad 5(38.5)$

(30.0)

(40.0)

$11(40.7) \quad 5(38.5)$

8 (29.6) $\quad 3(23.1)$

1.90

(27.5)

0.37

$16(59.3) \quad 9(75.0)$

(62.5)

$5(18.5 \%) \quad 5(38.5 \%) \quad 1.86$ 
Panic Disorder, $\quad 8$

n (\%)

(20.0\%)

$6(22.2 \%) \quad 2(15.4 \%)$

Agoraphobia, $\mathrm{n} \quad 10$

(\%)

(25.0\%)

$4(14.8 \%) \quad 6(46.2 \%)$

$4.60 *$

Obsessive

9

Compulsive

Disorder, n (\%)

(22.5\%)

$6(22.2 \%) \quad 3(23.1 \%)$

Generalized

7

Anxiety

Disorder, n (\%)

(17.5\%)

$3(11.1 \%) \quad 4(30.8 \%)$

DASS

10.8

0.37

Depression, $M$

$10.6(5.3) \quad 11.3(4.0)$

(SD)

DASS Anxiety,

8.2

1.8

$M$ (SD)

(4.4)

$7.3(4.9) \quad 10.0(2.2)$

Baseline CAPS, $\quad 71.8$

$72.7(19.7) \quad 69.9(12.5)$

0.46

$M$ (SD)

(17.6)

2.35

.01 
Baseline Fear,

$M$ (SD)

Posttreatment

Fear, $M$ (SD)

Baseline

Dysphoria, $M$

(SD)

Posttreatment

Dysphoria, $M$

(SD)
40.0

13.8

(11.4)

31.4

17.1
$40.2(9.7) \quad 39.6(9.8)$

0.17

8.78**

$7.5(5.4) \quad 27.1(8.7)$

0.41

31.8 (12.7) $\quad 30.3$ (6.3)

$4.89 * *$

$9.7(8.1) \quad 23.6(9.2)$

$*$ indicates a significant difference at $p<.05 . * *$ indicates a significant difference at $p<0.001$.

Table 2. Summary of voxelwise region of interest analyses for pre-treatment neural activation and connectivity associations with reduction in PTSD severity. Correlations between percentage improvement in clinician administered PTSD severity scores (overall CAPS, and individual factor scores [Fear and Dysphoria] and pre-treatment activation of Regions of Interest are reported significant at $p_{F W E}<0.05$ below. (L-left, pgACC - pregenual anterior cingulate cortex).

\begin{tabular}{|c|c|c|c|c|c|c|}
\hline Emotion & Direction & Brain Region & $\begin{array}{l}\text { Cluster } \\
\text { size in } \\
\text { voxels }\end{array}$ & $\begin{array}{c}\text { Peak MNI } \\
\text { Coordinates } \\
(\mathrm{X}, \mathrm{Y}, \mathrm{Z})\end{array}$ & $\begin{array}{c}\text { Peak } \\
\text { z- } \\
\text { score }\end{array}$ & $\begin{array}{c}p \text {-value } \\
\text { (FWE } \\
\text { corrected) }\end{array}$ \\
\hline \multicolumn{7}{|c|}{ Supraliminal emotions } \\
\hline \multicolumn{7}{|c|}{ Correlation with reduction in overall symptom score } \\
\hline $\begin{array}{l}\text { Sad vs } \\
\text { Neutral }\end{array}$ & Positive & L Insula & 9 & $-42,2,0$ & 3.86 & 0.036 \\
\hline $\begin{array}{l}\text { Sad vs } \\
\text { Neutral }\end{array}$ & Negative & $\begin{array}{c}\text { L Insula <> } \\
\text { pgACC }\end{array}$ & 12 & 6, 38, 4 & 3.99 & 0.011 \\
\hline \multicolumn{7}{|c|}{ Correlation with reduction in Fear factor score } \\
\hline
\end{tabular}

This article is protected by copyright. All rights reserved. 


\begin{tabular}{|c|c|c|c|c|c|c|}
\hline $\begin{array}{l}\text { Sad vs } \\
\text { Neutral }\end{array}$ & Positive & L Insula & 3 & $-44,2,4$ & 3.82 & 0.042 \\
\hline $\begin{array}{l}\text { Fear vs } \\
\text { Neutral }\end{array}$ & Positive & L Insula & 55 & $-42,8,8$ & 4.04 & 0.009 \\
\hline $\begin{array}{l}\text { Sad vs } \\
\text { Neutral }\end{array}$ & Negative & $\begin{array}{c}\text { L Insula <> } \\
\text { pgACC }\end{array}$ & 10 & $4,40,-2$ & 3.85 & 0.019 \\
\hline \multicolumn{7}{|c|}{ Correlation with reduction in Dysphoria factor score } \\
\hline $\begin{array}{l}\text { Sad vs } \\
\text { Neutral }\end{array}$ & Negative & $\begin{array}{c}\text { L Insula <> } \\
\text { pgACC }\end{array}$ & 12 & $6,38,4$ & 3.99 & 0.011 \\
\hline
\end{tabular}

Table 3. Responders and non-responders versus controls in activation and connectivity for supraliminal emotion processing. Extracted betas for significant activation and connectivity results for each contrast are included. Brain measures were included in an ANOVA analysis comparing all groups with Bonferroni corrected posthoc comparisons conducted between each group. (L-left, pgACC - pregenual anterior cingulate cortex, $\mathrm{HC}$ - Healthy Controls).

\begin{tabular}{|c|c|c|c|c|c|c|c|c|c|c|c|c|}
\hline \multirow{2}{*}{$\begin{array}{c}\text { Br } \\
\text { ain } \\
\text { Re } \\
\text { gio } \\
\text { n }\end{array}$} & \multirow[t]{2}{*}{$\begin{array}{c}\text { Corre } \\
\text { lation } \\
\text { varia } \\
\text { ble }\end{array}$} & \multirow{2}{*}{$\begin{array}{c}\text { Resp } \\
\text { onder } \\
\mathbf{S} \\
\\
\text { Mean }\end{array}$} & $\begin{array}{c}\begin{array}{c}\text { Non- } \\
\text { Resp } \\
\text { onder } \\
\mathbf{s}\end{array} \\
\text { Mean }\end{array}$ & $\begin{array}{l}\text { Hea } \\
\text { lthy } \\
\text { Con } \\
\text { trol } \\
\text { S } \\
\\
\end{array}$ & \multicolumn{2}{|c|}{$\begin{array}{c}\text { Respon } \\
\text { ders vs } \\
\text { Non- } \\
\text { Respon } \\
\text { ders vs } \\
\text { HC }\end{array}$} & \multicolumn{2}{|c|}{$\begin{array}{l}\text { Non- } \\
\text { Responde } \\
\text { rs vs } \\
\text { Responde } \\
\text { rs }\end{array}$} & \multicolumn{2}{|c|}{$\begin{array}{l}\text { Responde } \\
\text { rs vs HC }\end{array}$} & \multicolumn{2}{|c|}{$\begin{array}{c}\text { Non- } \\
\text { Responde } \\
\text { rs vs HC }\end{array}$} \\
\hline & & & $\begin{array}{l}\text { Mean } \\
(S D)\end{array}$ & $\begin{array}{c}\text { Mea } \\
n \\
(S D)\end{array}$ & $F$ & $\begin{array}{c}P- \\
v a \\
l u \\
e\end{array}$ & $\begin{array}{c}\text { Mea } \\
n \\
\text { Diffe } \\
\text { renc } \\
e\end{array}$ & $\begin{array}{l}P- \\
\text { val } \\
\text { ue }\end{array}$ & $\begin{array}{c}\text { Mea } \\
n \\
\text { Diffe } \\
\text { renc } \\
e\end{array}$ & $\begin{array}{l}P- \\
v a \\
l u \\
e\end{array}$ & $\begin{array}{c}\text { Mea } \\
n \\
\text { Diffe } \\
\text { renc } \\
e\end{array}$ & $\begin{array}{l}P- \\
v a \\
l u \\
e\end{array}$ \\
\hline
\end{tabular}

\section{Supraliminal}

\section{Sad vs Neutral}

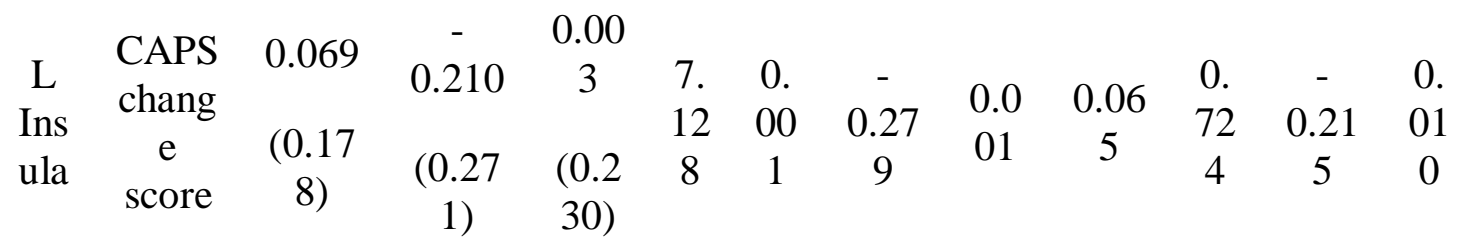

This article is protected by copyright. All rights reserved. 
$\begin{array}{ccccccccccccc} & \text { Fear } & 0.095 & - & 0.01 & & & & & & & & \\ \text { L } & \text { chang } & & 0.173 & 7 & 8 . & 0 . & - & <0 . & 0.07 & 0 . & - & 0 . \\ \text { Ins } & \text { e } & (0.15 & & & 13 & 00 & 0.26 & 00 & 8 & 35 & 0.19 & 01 \\ \text { ula } & \text { score } & 9) & (0.24 & (0.2 & 1 & 1 & 8 & 1 & & 0 & 0 & 0\end{array}$

L

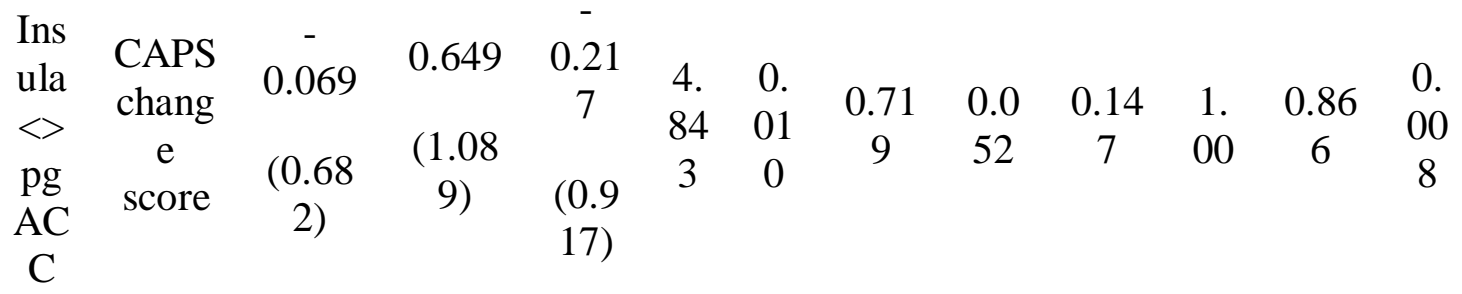

L C

L

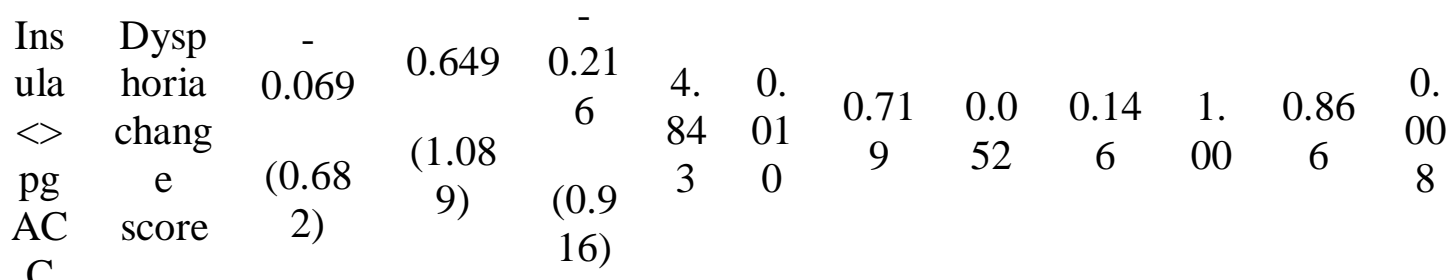

\section{Fear vs Neutral}

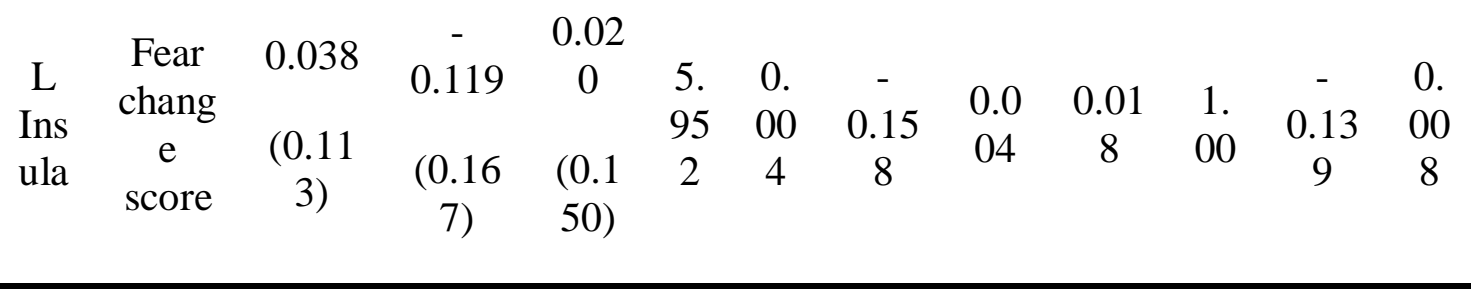

This article is protected by copyright. All rights reserved. 


\section{University Library}

\section{- M I I N E R VA \\ A gateway to Melbourne's research publications}

Minerva Access is the Institutional Repository of The University of Melbourne

Author/s:

Bryant, RA;Erlinger, M;Felmingham, K;Malhi, GS;O'Donnell, ML;Williams, LM;Korgaonkar, MS

Title:

Differential neural predictors of treatment response for fear and dysphoric features of posttraumatic stress disorder

Date:

2020-06-24

Citation:

Bryant, R. A., Erlinger, M., Felmingham, K., Malhi, G. S., O'Donnell, M. L., Williams, L. M. \& Korgaonkar, M. S. (2020). Differential neural predictors of treatment response for fear and dysphoric features of posttraumatic stress disorder. DEPRESSION AND ANXIETY, 37 (10), pp.1026-1036. https://doi.org/10.1002/da.23061.

Persistent Link:

http://hdl.handle.net/11343/275944 